\title{
Association of vitamin D receptor and CYP2R1 mRNA expression with pulmonary tuberculosis
}

Hong-Miao Li ${ }^{1}$, Ye Li ${ }^{1}$, Gen-You Zhang ${ }^{1}$, Si-Jiu Shi ${ }^{1}$, Tian-Ping Zhang ${ }^{2}$

${ }^{1}$ Anhui Chest Hospital (Anhui Provincial TB Institute), China

${ }^{2}$ The First Affiliated Hospital of University of Science and Technology of China, China

Submitted: 23 June 2020

Accepted: 4 October 2020

Arch Med Sci

DOI: https://doi.org/10.5114/aoms.2020.100763

Copyright () 2020 Termedia \& Banach

\section{Abstract}

Introduction: The vitamin D metabolic pathway has been shown to play a pivotal role in the pathogenesis of pulmonary tuberculosis (PTB), and vitamin $D$ receptor (VDR) and CYP2R1 gene variation are known to affect vitamin D status. Hence, this study aimed to evaluate VDR and CYP2R1 mRNA expression in peripheral blood mononuclear cells (PBMCs) in PTB patients.

Material and methods: We measured VDR and CYP2R1 mRNA levels in 75 PTB patients and 63 healthy controls by quantitative real-time reverse transcription polymerase chain reaction ( $q R T-P C R)$. The associations of VDR and CYP2R1 mRNA levels with clinical characteristics and laboratory indexes of PTB patients were also examined in this study.

Results: Compared to healthy controls, the VDR mRNA level was significantly higher, and the CYP2R1 mRNA level was significantly lower in PBMCs from PTB patients ( $p=0.047, p=0.008$, respectively). The CYP2R1 mRNA level in PTB patients with drug-resistant, unilateral tuberculosis foci was significantly higher than that in PTB patients without these clinical characteristics ( $p=0.005, p=0.048$, respectively). In addition, our results demonstrated that the VDR mRNA expression level was positively correlated with erythrocyte sedimentation rate $(E S R)(p=0.045)$, while the CYP2R1 mRNA level was negatively correlated with ESR in PTB patients $(p=0.020)$.

Conclusions: Altered VDR and CYP2R1 mRNA expression levels among PTB patients suggest their involvement in this disease.

Key words: vitamin D receptor, CYP2R1, pulmonary tuberculosis, PBMCs.

\section{Introduction}

Tuberculosis (TB) is still one of the most important deadly infectious diseases worldwide and is also an ongoing and major health problem. The latest World Health Organization (WHO) figures show that there were approximately 10.0 million new incident TB patients globally in 2018. Of them, 0.87 million cases were from China [1]. The main pathogen of TB is Mycobacterium tuberculosis (MTB), an intracellular Gram-positive bacterium, and this intracellular bacterium can escape the host immune system and remain dormant in macrophages until the immune system is suppressed. Cell-mediated immunity is essential for inhibiting MTB infection, regulating the first defense against MTB, and has an important role in the development of TB [2, 3]. Previous evidence has shown that only $10 \%$ of MTB infections eventually progress to active TB, suggesting that genetic factors, immune status,

\author{
Corresponding author: \\ Tian-Ping Zhang PhD \\ The First Affiliated Hospital \\ of University of Science and \\ Technology of China \\ 17 Lujiang Road, Hefei \\ Anhui 230001, China \\ E-mail: \\ zhangtianping@ustc.edu.cn
}


and environmental factors are closely related to the pathophysiology of TB [4-6].

Vitamin $D$ deficiency is caused by multiple environmental factors, including reduced access to dietary calciferol and decreased synthesis of cholecalciferol in skin, and is characterized by decreased serum 25-hydroxyvitamin D levels, the main circulating metabolite of vitamin D [7]. Recently, increasing research has investigated the roles of the vitamin D metabolic pathway in TB. In vitro studies showed that 1,25-dihydroxyvita$\min \mathrm{D}_{3}\left(1,25(\mathrm{OH})_{2} \mathrm{D}_{3}\right)$, the active form of vitamin $\mathrm{D}$, could enhance innate immunity by increasing antimicrobial peptide expression and inducing autophagy in infected cells, thereby limiting the intracellular growth of MTB in macrophages [8]. Moreover, vitamin D deficiency is much more prevalent among TB patients, and serum vitamin $D$ levels are negatively correlated with TB severity [9-11]. Vitamin D is well known to exert its biological functions through binding to the vitamin $D$ receptor (VDR). Panda et al. reported that after binding with vitamin $D, V D R$ formed a heterodimer with the retinoid $X$ receptor, and the heterodimer bound to vitamin $D$ response elements in the promoter region of vitamin $D_{3}$-regulated genes to mediate the interaction of regulatory proteins, hence regulating transcription [12]. Genome-wide association studies have suggested that $25(\mathrm{OH}) \mathrm{D}$ levels are related to genetic factors $[13,14]$. One of the few genes associated with serum $25(\mathrm{OH}) \mathrm{D}$ levels was CYP2R1, which is a member of the cytochrome P450 superfamily of monooxygenases [15]. CYP2R1 encodes the main liver vitamin $D$ 25 -hydroxylase, which might convert vitamin $D$ into $25(\mathrm{OH}) \mathrm{D}$.

Therefore, it seems that VDR and CYP2R1 might be involved in TB development. In addition, the potential associations between mutations in the $V D R$ and CYP2R1 genes and TB have been as sessed in many studies [16-18]. To our knowledge, no studies have been conducted to assess VDR or CYP2R1 mRNA expression levels in peripheral blood mononuclear cells (PBMCs) in pulmonary TB (PTB) patients. Therefore, our study aimed to analyze the differences in VDR and CYP2R1 mRNA expression levels between PTB patients and healthy controls and to assess the relationship between VDR and CYP2R1 mRNA levels and the main clinical features of PTB patients in a Chinese population.

\section{Material and methods}

\section{Study object}

A total of 75 diagnosed TB patients and 63 healthy controls were included in the study. Patients with PTB were recruited from the Depart- ment of Tuberculosis at Anhui Chest Hospital (Anhui Provincial TB Institute) from June to October 2019. All PTB patients were diagnosed by specialist physicians according to the following criteria: suspicious clinical symptoms, chest radiography, sputum and/or bronchoalveolar lavage fluid MTB culture, microscopy for acid fast bacilli (AFB), and effect of anti-TB treatment. Among these PTB patients, individuals with a history of cancer, hepatitis, immune-compromised conditions, and HIV were excluded. Healthy controls who had normal chest radiographic results and no history of TB, diabetes, malignant tumor, or HIV were enrolled in the same area. After receiving the study consent forms, we began to collect peripheral blood samples and information from each participant. We collected the main clinical and laboratory parameters of PTB patients from their medical records, including sputum smear, chest radiography, complications, total bilirubin (TBIL), aspartate aminotransferase (AST), alanine aminotransferase (ALT), and erythrocyte sedimentation rate (ESR). This study was approved by the Ethics Committee of Anhui Chest Hospital (K2020-005).

\section{Measurement of vitamin $D$ receptor and CYP2R1 mRNA expression levels}

We measured the VDR and CYP2R1 mRNA expression levels in PBMCs using quantitative realtime reverse transcription polymerase chain reaction (qRT-PCR) with SYBR Green (SYBR Premix Ex Taq II, Takara Bio Inc., Japan). We extracted $5 \mathrm{ml}$ of anticoagulated peripheral blood through the EDTA anticoagulant tube from each subject and isolated PBMCs from peripheral blood through Ficoll-Hypaque density gradient centrifugation. To ensure the purity and identification of PBMCs, the following measures were taken in the experimental process. First, all collected peripheral blood was sent to the laboratory within 4 hours for PBMC extraction. Second, an equal volume of peripheral blood sample and lymphocyte separation fluid were added to each centrifuge tube. Third, the PBMC layer was washed twice with PBS. Finally, the extracted PBMCs were stored in enzyme-free EP tubes. Then, the total RNA in PBMCs was extracted using TRIzol reagent, and the purity and concentrations of total RNA were measured using a NanoDrop 2000 spectrophotometer. Total RNA was reverse-transcribed into cDNA, which was used for PCR experiments with the PrimeScript RT reagent Kit.

The PCR experiment in this study was conducted on a QuantStudio 12K Flex Real-Time PCR System (Applied Biosystems, Foster City, CA, USA). The relative expression levels of VDR and CYP2R1 were estimated using $2^{-\Delta \Delta C t}$ normalized to the housekeeping gene $\beta$-actin in the same 
sample. The primer sequences were as follows: 5'-CACGAAACTACCTTCAACTCC-3' (sense primer) and 5'-CATACTCCTGCTTGCTGATC-3' (antisense primer) for $\beta$-actin, 5'-ATCTGCATCGTCTCCCCAGAT-3' (sense primer) and 5'-AGCGGATGTACGTCTGCAGTG-3' (antisense primer) for VDR, and 5'-GACAGACCATGCCTTCCTTTA-3' (sense primer) and 5'-ATCGTCTGTGATCAACC CATC-3' (antisense primer) for CYP2R1.

\section{Statistical analysis}

The VDR and CYP2R1 mRNA levels were expressed as the median value and interquartile range. The nonparametric Mann-Whitney $U$ test was used to analyze differences between VDR and CYP2R1 mRNA levels of PTB patients and healthy controls, as well as the VDR and CYP2R1 mRNA levels of PTB patients with and without multiple clinical characteristics. Spearman's rank correlation coefficient test was used to test for the correlations between VDR and CYP2R1 mRNA levels and the laboratory indexes in PTB patients. A $p$-value of $<0.05$ was considered statistically significant. SPSS 23.0 software was used for the statistical analysis.

\section{Results}

The average age of PTB patients was 44.73 \pm 17.16 years, including 50 males and 25 females, while the healthy controls consisted of 45 males and 18 females, with a mean age of $44.68 \pm 10.28$ years. There were no significant differences regarding age and gender distribution between PTB patients and healthy controls (both $p>0.05$ ).

\section{Vitamin D receptor and CYP2R1 mRNA levels in peripheral blood mononuclear cells from pulmonary tuberculosis patients and controls}

The VDR and CYP2R1 mRNA expression levels in PBMCs from PTB patients and healthy controls are presented in Figure 1 . The results suggested that, compared to healthy controls, the VDR mRNA expression level was significantly higher in PBMCs from PTB patients ( $p=0.047$ ). In addition, the CYP2R1 mRNA level in PTB patients was significantly lower than that in healthy controls $(p=0.008)$.

\section{Relationship of vitamin D receptor} and CYP2R1 mRNA levels with clinical characteristics and laboratory indexes in pulmonary tuberculosis patients

Among PTB patients, 31 (41.33\%), 49 (65.33\%) and $17(22.67 \%)$ patients were diagnosed with active PTB patients, treatment-naive PTB patients, and drug-resistant PTB patients at the same
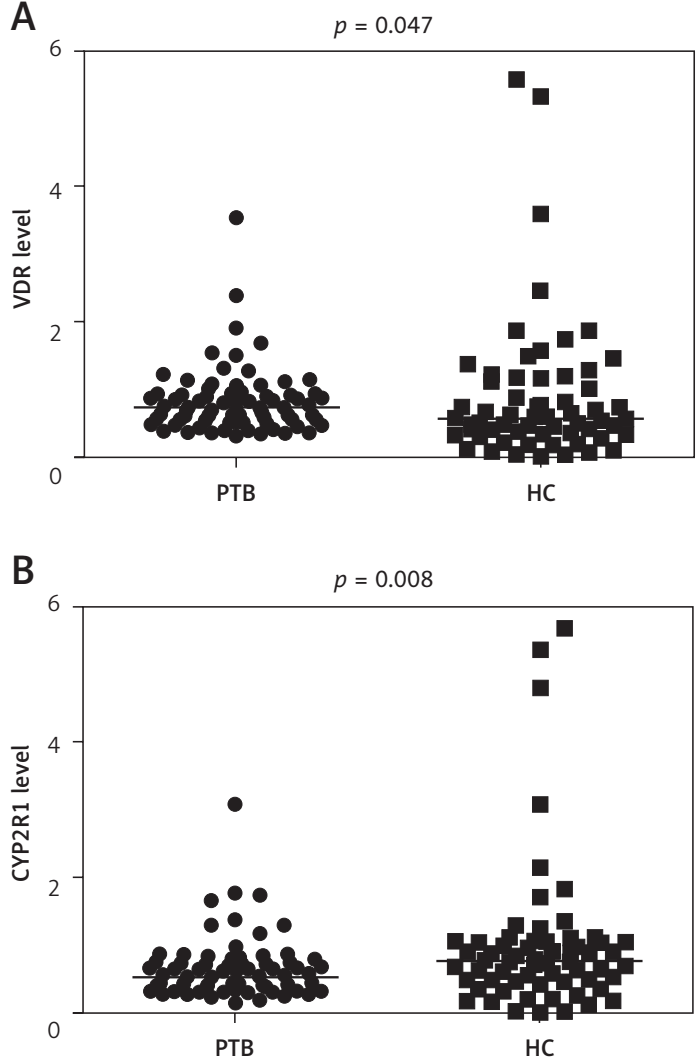

Figure 1. Comparison of vitamin D receptor (VDR) (A), CYP2R1 (B) mRNA level in peripheral blood mononuclear cells between pulmonary tuberculosis (PTB) patients and healthy controls ( $\mathrm{HC})$

time, respectively. In addition, other complications and clinical characteristics of PTB patients were sputum smear-positive (41.33\%), pulmonary cavity (36.00\%), unilateral tuberculosis foci (25.33\%), diabetes (20.00\%), extrapulmonary tuberculosis (13.33\%), and liver injury (9.33\%). We also explored the associations between VDR and CYP2R1 mRNA levels and the above clinical data in PTB (Table I). The CYP2R1 level was significantly higher in PTB patients with drug resistance compared with PTB patients without drug resistance $(p=0.005)$. In comparison to PTB patients with unilateral tuberculosis foci, the CYP2R1 mRNA level was significantly lower in those patients with bilateral tuberculosis foci ( $p=0.048$ ). However, no statistical association between VDR level and these clinical characteristics was found (Table I).

The correlations of VDR and CYP2R1 mRNA levels with laboratory indicators, including ESR, TBIL, ALT, and AST, of PTB patients were also analyzed (Table II). Our results demonstrated that the VDR mRNA expression level was positively correlated with ESR ( $p=0.045)$, while the CYP2R1 mRNA level was negatively correlated with ESR in PTB patients $(p=0.020)$. 
Table I. Correlation between vitamin D receptor (VDR) and CYP2R1 expression levels and some clinical features in pulmonary tuberculosis patients

\begin{tabular}{|c|c|c|c|c|c|c|}
\hline Group & $+/-$ & Number & VDR level & $P$-value & CYP2R1 level & $P$-value \\
\hline \multirow[t]{2}{*}{ Treatment-naïve patients } & + & 49 & $0.760(0.611,0.933)$ & \multirow[t]{2}{*}{0.764} & $0.477(0.321,0.664)$ & \multirow[t]{2}{*}{0.124} \\
\hline & - & 26 & $0.657(0.505,0.965)$ & & $0.618(0.392,0.810)$ & \\
\hline \multirow[t]{2}{*}{ Drug-resistant patients } & \multirow{2}{*}{$\begin{array}{l}+ \\
-\end{array}$} & 17 & $0.683(0.523,0.941)$ & \multirow[t]{2}{*}{0.552} & $0.599(0.420,0.810)$ & \multirow[t]{2}{*}{0.005} \\
\hline & & 58 & $0.824(0.553,0.942)$ & & $0.369(0.313,0.534)$ & \\
\hline \multirow[t]{2}{*}{ Liver injury } & + & 7 & $0.623(0.564,0.876)$ & \multirow[t]{2}{*}{0.636} & $0.709(0.537,0.840)$ & \multirow[t]{2}{*}{0.308} \\
\hline & - & 68 & $0.740(0.514,0.950)$ & & $0.507(0.359,0.732)$ & \\
\hline \multirow[t]{2}{*}{ Diabetes } & + & 15 & $0.818(0.560,0.937)$ & \multirow[t]{2}{*}{0.791} & $0.452(0.308,0.692)$ & \multirow[t]{2}{*}{0.289} \\
\hline & - & 60 & $0.683(0.514,0.965)$ & & $0.538(0.378,0.758)$ & \\
\hline \multirow[t]{2}{*}{ Sputum smear-positive ${ }^{a}$} & + & 31 & $0.775(0.560,0.955)$ & \multirow[t]{2}{*}{0.491} & $0.467(0.308,0.726)$ & \multirow[t]{2}{*}{0.217} \\
\hline & - & 41 & $0.682(0.518,0.958)$ & & $0.557(0.394,0.834)$ & \\
\hline \multirow[t]{2}{*}{ Unilateral tuberculosis foci } & + & 19 & $0.682(0.387,1.063)$ & \multirow[t]{2}{*}{0.401} & $0.742(0.418,0.874)$ & \multirow[t]{2}{*}{0.048} \\
\hline & - & 56 & $0.753(0.566,0.936)$ & & $0.484(0.359,0.685)$ & \\
\hline \multirow[t]{2}{*}{ Pulmonary cavity } & + & 27 & $0.728(0.560,0.932)$ & \multirow[t]{2}{*}{0.825} & $0.554(0.322,0.742)$ & \multirow[t]{2}{*}{0.921} \\
\hline & - & 48 & $0.730(0.502,0.966)$ & & $0.520(0.369,0.776)$ & \\
\hline \multirow[t]{2}{*}{ Extrapulmonary tuberculosis } & + & 10 & $0.744(0.562,0.946)$ & \multirow[t]{2}{*}{0.334} & $0.539(0.368,0.752)$ & \multirow[t]{2}{*}{0.427} \\
\hline & - & 65 & $0.602(0.444,0.943)$ & & $0.506(0.298,0.711)$ & \\
\hline \multirow[t]{2}{*}{ Active PTB } & + & 31 & $0.731(0.560,0.940)$ & \multirow[t]{2}{*}{0.796} & $0.467(0.322,0.714)$ & \multirow[t]{2}{*}{0.282} \\
\hline & - & 44 & $0.703(0.514,0.961)$ & & $0.548(0.389,0.819)$ & \\
\hline
\end{tabular}

+/-: with/without; median (interquartile range); ' 50 me of the study subjects' data are missing.

Table II. Correlation between vitamin D receptor (VDR) and CYP2R1 mRNA levels and erythrocyte sedimentation rate (ESR), total bilirubin (TBIL), alanine transaminase (ALT), aspartate aminotransferase (AST) of pulmonary tuberculosis patients

\begin{tabular}{|lcccc|}
\hline \multirow{2}{*}{$\begin{array}{l}\text { Clinical } \\
\text { parameters }\end{array}$} & \multicolumn{2}{c}{ VDR level } & \multicolumn{2}{c|}{ CYP2R1 level } \\
\cline { 2 - 5 } & $r_{\mathrm{s}}$ & $P$-value & $r_{\mathrm{s}}$ & $P$-value \\
\hline ESR & 0.244 & 0.045 & -0.281 & 0.020 \\
\hline TBIL & -0.036 & 0.761 & -0.016 & 0.895 \\
\hline ALT & -0.198 & 0.089 & 0.104 & 0.375 \\
\hline AST & 0.099 & 0.396 & -0.182 & 0.117 \\
\hline
\end{tabular}

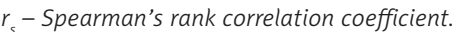

\section{Discussion}

Because of the modulatory impact of vitamin D on the innate immune system, such as the response to the MTB pathogen, many researchers have tried to explore the specific role of genetic variations in the vitamin $D$ pathway, including $V D R, C Y P 2 R 1$, and vitamin $\mathrm{D}$ expression levels, in the pathophysiology of PTB [19-21]. Several studies showed that low vitamin $D$ levels could lead to the relapse of PTB patients and might mediate an adverse effect on clinical improvement [22]. Here, we first found increased VDR mRNA levels and decreased CYP2R1 mRNA levels in PBMCs from PTB patients, and CYP2R1 mRNA levels were significantly related to several clinical characteristics of PTB patients.

Previous studies have verified the role of vitamin $D$ in regulating the host immune response through VDR activity in activated human monocytes, the ability of $1,25(\mathrm{OH})_{2} \mathrm{D}_{3}$ to inhibit $\mathrm{T}$ cell proliferation, and the ability of pathogen-activated macrophages to produce $1,25(\mathrm{OH})_{2} \mathrm{D}_{3}[23,24]$. As one of the nuclear receptors of transcription factors, VDR can bind to the response elements of DNA and form heterodimers with the retinoic acid receptor (RXR), thereby affecting downstream gene expression [25]. One of the downstream target genes encoded cathelicidin, known as a cationic antimicrobial peptide, which can penetrate cellular membranes to result in the direct lysis of mycobacterium. In addition, $1,25(\mathrm{OH})_{2} \mathrm{D}_{3}$ has been reported to be associated with IFN- $\gamma$ to control the proliferation of MTB by human monocytes $[23,26]$. VDR variants were also found to lead to diminished function of vitamin $D$ and were involved in PTB development. Hu et al. ob- 
served that the A alleles of VDR rs11574143 and rs11168287 increased the risk of PTB, and the VDR rs11574079 A allele provided a protective effect against PTB [27]. A meta-analysis showed that the VDR 1544410 polymorphism might be related to an increased risk of PTB in Asians [28]. In addition, a previous study found that VDR mRNA expression in MTB-stimulated macrophage culture was significantly higher than that in unstimulated cultures [29]. Similarly, our results demonstrated that the expression level of VDR mRNA was significantly increased in PBMCs from PTB patients. This result was also in accordance with another study in which upregulated expression of VDR was observed in patients with asthma [30]. Based on the findings of this study, we speculated that MTB might evade the host immune system by altering the expression of genes such as VDR, but further functional studies are needed to verify this hypothesis. Our results also suggested that the VDR mRNA expression level was positively correlated with ESR in PTB patients.

Vitamin $D$ is activated through two enzymatic steps. Vitamin $D_{3}$ is converted in the liver by hepatic 25-hydroxylases (CYP2R1) to 25-hydroxycholecalciferol (25-hydroxyvitamin $\mathrm{D}_{3},\left[25(\mathrm{OH}) \mathrm{D}_{3}\right]$ ). Then, $25(\mathrm{OH}) \mathrm{D}_{3}$ is metabolized to 1,25-dihydroxycholecaciferol $\left(1,25[\mathrm{OH}]_{2} \mathrm{D}_{3}\right)$ in the kidney by $1 \alpha$-hydroxylase (CYP27B1) [31]. Because of the role of CYP2R1 in the metabolism of vitamin D, CYP2R1 might also be associated with the pathogenesis of PTB. CYP2R1 gene variation is known to affect vitamin D status, and several studies have investigated the association between CYP2R1 SNPS and PTB susceptibility. Junaid et al. explored the main effects of SNPS (rs2060793, rs10500804 and rs10766197) in CYP2R1 on susceptibility to PTB in the Pakistan population; however, no significant result was found [21]. Similarly, Sadykov et al. also found that there was no significant relationship between CYP2R1 rs10741657 and PTB risk in the Kazakhstan population [18]. In the present study, we directly detected the expression level of CYP2R1 mRNA in PBMCs of PTB patients. We assumed that lower CYP2R1 levels might contribute to vitamin D deficiency in PTB patients, which could provide a potential treatment strategy for PTB patients. In addition, we also found that CYP2R1 mRNA levels were significantly increased in PTB patients with drug-resistant and unilateral tuberculosis foci.

At present, the diagnosis of PTB remains a great challenge, although Murdaca et al. found that multiple miRNAs played crucial roles in the pathologic mechanisms of PTB and had been proposed as diagnostic biomarkers and therapeutic targets for future treatments [32]. In our study, the results suggested that VDR and CYP2R1 were involved in the pathogenesis of PTB and might be used for PTB diagnosis. Moreover, we also explored the potential relationship between VDR and CYP2R1 levels and a variety of clinical characteristics of PTB patients. However, there were some limitations in our study. First, the sample size of the study might be insufficient, which could affect the power of the study. Second, due to the lack of information, this study did not analyze the potential influence of clinical medication, disease activity, and other factors on VDR and CYP2R1 mRNA expression. Hence, further large, functional studies will be needed to further explore the immune-regulating effect of the vitamin D pathway in PTB.

In conclusion, our results provide evidence that VDR and CYP2R1 mRNA levels are associated with the development of PTB. In addition, VDR and CYP2R1 mRNA levels were related to some clinical characteristics, including drug resistance, tuberculosis foci, and ESR, in PTB patients. VDR and CYP2R1 should be studied further for consideration of potential biomarkers for PTB diagnosis.

\section{Acknowledgement}

Hong-Miao Li and Ye Li contributed equally to this work and should be considered co-first authors.

The Authors are very grateful to Prof. Li Wei from the UCL School of Pharmacy for her critical review of this manuscript.

This research was funded by Anhui Provincial Natural Science Foundation (1908085QH368).

\section{Conflict of interest}

The authors declare no conflict of interest.

\section{References}

1. World Health Organization. 2019. Global tuberculosis report 2019. Available at: https://www.who.int/tb/publications/global_report/en/.

2. Silva-Ramírez B, Saenz-Saenz CA, Bracho-Vela LA, et al. Association between vitamin $D$ receptor gene polymorphisms and pulmonary tuberculosis in a Mexican population. Indian J Tuberc 2019; 66: 70-5.

3. Azad AK, Sadee W, Schlesinger LS. Innate immune gene polymorphisms in tuberculosis. Infect Immun 2012; 80: 3343-59.

4. Wang Y, Li HJ. A meta-analysis on associations between vitamin $\mathrm{D}$ receptor genetic variants and tuberculosis. Microb Pathog 2019; 130: 59-64.

5. O'Garra A, Redford PS, McNab FW, et al. The immune response in tuberculosis. Annu Rev Immunol 2013; 31: 475-527.

6. Cao Y, Wang X, Cao Z, et al. Genetic polymorphisms of MBL2 and tuberculosis susceptibility: a meta-analysis of 22 case-control studies. Arch Med Sci 2018; 14 : 1212-32.

7. Roizen JD, Long C, Casella A, et al. Obesity decreases hepatic 25-hydroxylase activity causing low serum 
25-hydroxyvitamin D.J Bone Miner Res 2019; 34: 1068-73.

8. Selvaraj P, Harishankar M, Afsal K. Vitamin D: immunomodulation and tuberculosis treatment. Can J Physiol Pharmacol 2015; 93: 377-84.

9. Gou X, Pan L, Tang F, et al. The association between vitamin D status and tuberculosis in children: a meta-analysis. Medicine (Baltimore) 2018; 97: e12179.

10. Brighenti S, Bergman P, Martineau AR. Vitamin D and tuberculosis: where next? J Intern Med 2018; doi: 10.1111/ joim. 12777.

11. Joo MH, Han MA, Park SM, et al. Vitamin D deficiency among adults with history of pulmonary tuberculosis in Korea based on a nationwide survey. Int J Environ Res Public Health 2017; 14: 399.

12. Panda S, Tiwari A, Luthra K, et al. Association of Fok1 VDR polymorphism with vitamin D and its associated molecules in pulmonary tuberculosis patients and their household contacts. Sci Rep 2019; 9: 15251.

13. Ahn J, Yu K, Stolzenberg-Solomon R, et al. Genome-wide association study of circulating vitamin D levels. Hum Mol Genet 2010; 19: 2739-45.

14. Anderson D, Holt BJ, Pennell CE, et al. Genome-wide association study of vitamin D levels in children: replication in the Western Australian Pregnancy Cohort (Raine) study. Genes Immun 2014; 15: 578-83.

15. Cheng JB, Levine MA, Bell NH, et al. Genetic evidence that the human CYP2R1 enzyme is a key vitamin D 25-hydroxylase. Proc Natl Acad Sci U S A 2004; 101: 7711-5.

16. Sinaga BY, Amin M, Siregar Y, et al. Correlation between vitamin D receptor gene FOKI and BSMI polymorphisms and the susceptibility to pulmonary tuberculosis in an Indonesian Batak-ethnic population. Acta Med Indones 2014; 46: 275-82.

17. Medapati RV, Suvvari S, Godi S, et al. NRAMP1 and VDR gene polymorphisms in susceptibility to pulmonary tuberculosisamong Andhra Pradesh population in India: a case-control study. BMC Pulm Med 2017; 17: 89.

18. Sadykov M, Azizan A, Kozhamkulov U, et al. Association of genetic variations in the vitamin D pathway with susceptibility to tuberculosis in Kazakhstan. Mol Biol Rep 2020; 47: 1659-66.

19. Huang SJ, Wang XH, Liu ZD, et al. Vitamin D deficiency and the risk of tuberculosis: a meta-analysis. Drug Des Devel Ther 2016; 11: 91-102.

20. Areeshi MY, Mandal RK, Wahid M, et al. Vitamin D receptor Apal (rs7975232) polymorphism confers decreased risk of pulmonary tuberculosis in overall and African population, but not in Asians: evidence from a metaanalysis. Ann Clin Lab Sci 2017; 47: 628-37.

21. Junaid K, Rehman A, Jolliffe DA, et al. Vitamin D deficiency associates with susceptibility to tuberculosis in Pakistan, but polymorphisms in VDR, DBP and CYP2R1 do not. BMC Pulm Med 2016; 16: 73.

22. Mehta S, Mugusi FM, Bosch RJ, et al. Vitamin D status and TB treatment outcomes in adult patients in Tanzania: a cohort study. BMJ Open 2013; 3: e003703.

23. Rook GA, Steele J, Fraher L, et al. Vitamin D3, gamma interferon, and control of proliferation of Mycobacterium tuberculosis by human monocytes. Immunology 1986; 57: 159-63.

24. Crowle AJ, Ross EJ, May MH. Inhibition by $1,25(\mathrm{OH}) 2-\mathrm{vi}-$ tamin D3 of the multiplication of virulent tubercle bacilli in cultured human macrophages. Infect Immun 1987; 55: 2945-50

25. Wang TT, Nestel FP, Bourdeau V, et al. Cuttingedge: 1,25-dihydroxyvitamin D3 is a direct inducer of antimicrobial peptide gene expression. J Immunol 2004; 173:
2909-12.

26. Liu PT, Stenger S, Li H, et al. Toll-like receptor triggering of a vitamin D-mediated human antimicrobial response. Science 2006; 311: 1770-3.

27. $\mathrm{Hu} \mathrm{Q}$, Chen Z, Liang G, et al. Vitamin D receptor gene associations with pulmonary tuberculosis in a Tibetan Chinese population. BMC Infect Dis 2016; 16: 469.

28. Areeshi MY, Mandal RK, Dar SA, et al. A reappraised metaanalysis of the genetic association between vitamin $D$ receptor I (rs1544410) polymorphism and pulmonary tuberculosis risk. Biosci Rep 2017; 37: BSR20170247.

29. Selvaraj P, Prabhu Anand S, Harishankar M, et al. Plasma 1,25 dihydroxy vitamin D3 level and expression of vitamin $D$ receptor and cathelicidin in pulmonary tuberculosis. J Clin Immunol 2009; 29: 470-8.

30. Chary AV, Hemalatha R, Murali MV, et al. Association of T-regulatory cells and CD23/CD21 expression with vitamin $D$ in children with asthma. Ann Allergy Asthma Immunol 2016; 116: 447-54.

31. Hewison M, Burke F, Evans KN, et al. Extra-renal 25-hydroxyvitamin D3-1alpha-hydroxylase in human health and disease. J Steroid Biochem Mol Biol 2007; 103: 316-21.

32. Murdaca G, Tonacci A, Negrini S, et al. Effects of antagomiRs on different lung diseases in human, cellular, and animal models. Int J Mol Sci 2019; 20: 3938. 